\title{
INVESTIGACIONES
}

\section{Análisis y Evaluación de las Competencias Genéricas en la Formación Inicial del Profesorado}

\author{
Analysis and Evaluation of Generic Competencies \\ in Initial Teacher Training \\ $M^{a}$ Isabel Amor Almedina ${ }^{a}$, Rocío Serrano Rodríguez ${ }^{b}$ \\ ${ }^{\text {a }}$ Facultad de Ciencias de la Educación. Universidad de Córdoba \\ Correo electrónico:m.amor@uco.es \\ ${ }^{\mathrm{b}}$ Facultad de Ciencias de la Educación. Universidad de Córdoba \\ Correo electrónico: rocio.serrano@uco.es
}

\section{RESUMEN}

El propósito de este estudio es evaluar el nivel de desarrollo de las competencias genéricas en los grados universitarios. La muestra se formó de 1243 estudiantes, 491 graduados y 351 profesores de 23 universidades españolas. Los análisis estadísticos empleados han sido los siguientes: Análisis Factorial de Confirmación (CFA) de la escala para comprobar la estructura factorial del instrumento y análisis descriptivos básicos, para conocer cuáles son los grupos de competencias más desarrolladas en cada uno de los grupos que conforman la muestra. Posteriormente, se realizan análisis de varianza para conocer las diferencias entre los grupos. Los resultados muestran que existen diferencias significativas entre los tres grupos, pero, en general, la competencia para trabajar en equipo es la mejor percibida por todos los participantes, en contraposición a la capacidad de comunicación en un idioma y la competencia tecnológica.

Palabras claves: competencias genéricas, formación inicial del profesorado, educación superior.

\section{ABSTRACT}

The objective of this study was to evaluate the level of development of generic competencies in university degree programs. The sample was composed of 1243 students, 491 graduates, and 351 teachers from 23 Spanish universities. The statistical analyses employed in this study included the following: We first carried out a Confirmatory Factor Analysis (CFA) of the scale to test the factorial structure of the instrument and basic descriptive analyses to determine which groups of competencies were most developed in each of the groups composing sample. Subsequently, an analysis of variance was performed to understand the differences between the groups. The results obtained demonstrate that there were significant differences between the three groups, but in general, teamwork was the most noted competency for all participants, as opposed to the ability to communicate in a language and technological competence.

Keywords: generic competencies, initial teacher training, higher education. 


\section{INTRODUCCIÓN}

La universidad del siglo XXI, como institución educativa, se enfrenta constantemente a los retos que supone preparar de forma adecuada a los estudiantes para la sociedad actual, basada en el conocimiento y en la proyección laboral. Por ello, es necesario que todas las estructuras educativas se centren en proporcionar una base formativa sólida, fundamentada en el desarrollo de unas competencias genéricas y transversales a cualquier plan de estudios o currículo (Le Boterf, 2001; Perrenoud, 1997; Whiddett \& Hollyforde, 2003), una serie de habilidades comunes a todos los títulos universitarios que capaciten al alumnado para afrontar todas las circunstancias de su vida personal, social y profesional, competencias que les permitan adaptarse a todas y cada una de las situaciones que así lo requieran. Para hacer frente a este desafío educativo y formativo, la enseñanza y el aprendizaje por competencias se ha convertido en una alternativa muy consistente a los métodos tradicionales utilizados hasta ahora (Bartram \& Roe, 2005; Prieto, 2008; Villa \& García, 2009), y difiere bastante del enfoque basado fundamentalmente en la adquisición de conocimientos y demandas esenciales en la concepción pedagógica y en el perfil del profesor y el rol del estudiante en este nuevo contexto (Elen et al., 2007; Novak, 2003; Rogof, 1999; Rust, Price \& O’Donovan, 2003; Struyven, Dochy \& Janssens, 2010).

El Proyecto Tuning Educacional Structures in Europe I (González \& Wagenaar, 2003) a nivel europeo determinó esta selección de competencias a partir de un estudio realizado con graduados, empleadores y académicos, y en el que se les solicitaba su opinión sobre el grado de desarrollo adquirido en las competencias y habilidades, durante los estudios universitarios y su importancia para su formación (González \& Wagenaar, 2003). Sus resultados permitieron clasificar treinta competencias genéricas divididas en tres categorías: instrumentales, interpersonales y sistémicas y son las que permiten a los titulados universitarios alcanzar mayores niveles de empleabilidad y ciudadanía:

1. Las competencias instrumentales, tal y como su nombre indica, tienen una función instrumental. Se identifican con capacidades de carácter cognitivo, metodológico, tecnológico y lingüístico que posibilitan un desenvolvimiento académico básico al estudiante universitario. Están relacionadas con las siguientes habilidades y destrezas (Zabalza, 2011):

- Habilidades cognoscitivas, capacidad para aprender y gestionar pensamientos e ideas.

- Capacidades metodológicas, para gestionar el ambiente: organizar el tiempo, tomar decisiones, resolver problemas y poner en práctica estrategias de aprendizaje.

- Habilidades tecnológicas relacionadas con la utilización de medios tecnológicos de la información y la comunicación.

2. Destrezas lingüísticas tales como la comunicación oral y escrita y el conocimiento de una segunda lengua. Las competencias interpersonales están dirigidas a facilitar la interacción y la cooperación social, son aquellas relacionadas con las habilidades comunicativas y críticas. Incluyen competencias relacionadas con las habilidades para relacionarse con los demás, trabajar en equipo, expresar los propios sentimientos y poseer un compromiso ético y social (Bartram \& Roe, 2005). Se subdividen en 
- Individuales: relativas a la capacidad de expresar los sentimientos, habilidades críticas y de autocrítica.

- Sociales: relacionadas con la capacidad de trabajar en equipo, o la expresión de compromiso social o ético.

3. Las competencias sistémicas requieren la adquisición de las anteriores como base (instrumentales e interpersonales), y permiten aproximarse a la realidad en su complejidad de relaciones y no como un conjunto de hechos aislados. Según Zabala y Arnau (2008), suponen una combinación de comprensión, sensibilidad y conocimiento permitiendo al individuo que todas las partes de un todo se relacionen y se agrupen. Este tipo de competencias incluyen habilidades y destrezas relacionadas con aprender de forma autónoma, desarrollar la creatividad y ser capaz de adaptarse a situaciones nuevas.

Rodríguez-Esteban (2007) afirma que actualmente la enseñanza universitaria manifiesta una creciente preocupación por desarrollar en la formación de sus estudiantes las competencias transversales o genéricas. En la sociedad actual, el mercado laboral y los propios estudiantes, cada vez más, demandan una enseñanza más alejada de los contenidos disciplinares y de la formación tradicional, basada principalmente en las competencias específicas propias de cada titulación (Rodríguez-Esteban \& Vieira, 2009). En relación con las competencias instrumentales, el conocimiento de un segundo idioma, y más concretamente del inglés, han adquirido una gran relevancia en el contexto universitario (European Language Council ${ }^{1}$ ). Para garantizar la movilidad y el intercambio de la comunidad universitaria entre, como mínimo, la Unión Europea, para ampliar sus horizontes formativos, lo que significa que un segundo idioma es una competencia muy necesaria. Asimismo, también existen muchas universidades que ofertan alguna parte de sus contenidos en inglés, consistiendo en uno de los indicadores fundamentales para el prestigio y la calidad de las mismas.

Por otro lado, la sociedad actual, conocida como la Sociedad de la Información o del Conocimiento (Bartram \& Roe, 2005), manifiesta claras diferencias con relación a sus precedentes. Anteriormente, en relación al perfil académico para asegurar el éxito, la estabilidad laboral y el estatus profesional y social, solamente era necesario contar con la formación recibida durante los distintos itinerarios académicos. Sin embargo, hoy en día este panorama ha cambiado radicalmente debido a que el mundo laboral requiere que los perfiles académicos y profesionales estén en constante renovación. Cada vez más, resulta imprescindible especializar el conocimiento y fomentar la educación continua, enmarcada dentro de una época y un saber que se enfoca hacia la plataforma digital, que define nuevas redes y patrones de transmisión y generación de conocimiento. Todo esto condiciona la validez y certeza de ciertos saberes, disciplinas y metodologías que anteriormente se consideraban como permanentes y duraderas. Bajo este orden de ideas, se debe resaltar que la profesión docente tampoco es ajena a esta situación, gracias al desarrollo tecnológico, el quehacer y la práctica docente que deben reorientarse hacia un nuevo paradigma que dé lugar a otras metodologías acordes con los retos que supone educar a la sociedad del siglo XXI.

https://europa.eu/european-union/about-eu/institutions-bodies/council-eu_en 


\section{MÉTODO}

\subsection{OBJETIVOS}

1. Evaluar las competencias docentes genéricas en los estudiantes, profesores y egresados de las titulaciones universitarias de Magisterio (especialidad en Educación Física), Grado de Educación Primaria (mención en Educación Física) y Grado de Ciencias de las Actividad Física y Deportiva.

2. Conocer cuáles son los grupos de competencias docentes genéricas más desarrolladas por dimensiones, en cada uno de los grupos que conforman la muestra.

3. Analizar las diferencias existentes entre los tres grupos.

\subsection{PARTICIPANTES}

La muestra estuvo compuesta por tres grupos poblacionales: estudiantes, docentes y egresados, pertenecientes a las titulaciones universitarias de Magisterio (especialidad en Educación Física), Grado de Educación Primaria (mención en Educación Física) y Grado de Ciencias de la Actividad Física y Deportiva, de 23 universidades españolas distribuidas en tres zonas: zona norte (Universidades de Valladolid, León, Burgos Cantabria, Salamanca, País Vasco y La Coruña), zona este (Universidades de Zaragoza, Lleida, Autónoma de Barcelona, Barcelona, Ramón Llull, Vic y Valencia) y zona centro, sur y Canarias (Universidades Autónoma de Madrid, La Laguna, Alcalá, Murcia, Católica de Murcia, Castilla la Mancha, Granada, Sevilla y Córdoba). La distribución de la muestra fue la siguiente: 1243 estudiantes, 351 docentes y 491 egresados.

Se estableció el muestreo aleatorio simple para la selección de la muestra tomando como población de referencia los datos facilitados por las secretarías de los centros partícipes en la investigación en el curso académico 2014/2015. Para determinar la pertenencia a los distintos grupos poblacionales se establecieron los siguientes criterios: a) en el caso de los estudiantes, estar matriculado en tercer curso con al menos tres asignaturas troncales; b) en el caso de los docentes, impartir docencia en al menos una asignatura de las titulaciones estudiadas; y en el caso de los egresados, haber finalizado sus estudios en los cinco años anteriores a la investigación.

\subsection{INSTRUMENTO}

Para la recogida de la información se ha utilizado un cuestionario estructurado elaborado dentro de un proyecto de investigación más amplio ${ }^{2}$, que consta de 12 preguntas (72 ítems) valoradas con una escala tipo Likert de cinco puntos en función del grado de acuerdo del enunciado, de 0 ("nada") a 5 ("muchísimo"). Para la elaboración de esta escala se tuvieron en cuenta escalas ya construidas y la bibliografía que apoya esta temática.

Este estudio se ha llevado a cabo dentro del proyecto de $\mathrm{I}+\mathrm{D}+\mathrm{i}$ : "Las competencias docentes en la formación inicial del profesorado de educación física". Convocatoria de noviembre de 2013 del Programa Estatal de Investigación, Desarrollo e Innovación Orientada a los Retos de la Sociedad, en el marco del Plan Estatal de Investigación Científica y Técnica y de Innovación 2013-2016. Referencia: EDU 2013-42024-R. Duración: 3 años (2014-2016). 
En una primera fase, tras una revisión bibliográfica y el análisis de trabajos previos relacionados con el tema, se recopiló un conjunto amplio de preguntas que fueron el punto de partida para los primeros diseños de las escalas con una versión de 89 preguntas. Este cuestionario fue utilizado en otros estudios recientes (Martínez, Castejón \& Santos, 2012; Muros \& Luis-Pascual, 2012; Ruíz-Gallardo, Ruíz \& Ureña, 2013). En una segunda fase, este primer borrador fue evaluado por 9 expertos en Didáctica de la Expresión Corporal y de la Educación Física. Con los datos de estas evaluaciones, fueron seleccionadas las preguntas más pertinentes por su relevancia (los ítems más relacionados con el objeto de estudio) y claridad (fácilmente comprensibles, con afirmaciones simples). En una tercera fase, se realizaron los primeros pretest con sujetos de características parecidas al objeto de estudio, para asegurar su comprensión. Tras la depuración de las preguntas que aportaban poca fiabilidad y las definidas de manera poco clara, se elaboró la escala final formada por 46 ítems divididos en tres secciones: competencias docentes de Educación Física, competencias docentes genéricas y competencias para la docencia de la Educación Física. Los ítems son similares en todos los cuestionarios, adaptando la redacción de los mismos en función del grupo poblacional a que va dirigido: estudiantes, profesorado y egresados.

Finalmente, de las 46 preguntas recogidas en dicho cuestionario se han seleccionado y analizado 18 ítems (Tabla 1), ya que son los que se corresponden con nuestro primer objetivo y que hacen referencia a la evaluación de las Competencias docentes genéricas o transversales, distribuidas en tres dimensiones: Instrumentales, Personales y Sistémicas, apoyadas por el Espacio Europeo de Educación Superior (EEES) y establecidas en el Proyecto Tuning Educacional Structures in Europe I (González \& Wagenaar, 2003).

Tabla 1. Ítems de la escala Evaluación de las Competencias Docentes

\begin{tabular}{|c|c|c|}
\hline Dimensiones & Ítem & Competencias \\
\hline \multirow{6}{*}{ Instrumentales } & 1 & Capacidad de análisis y síntesis \\
\hline & 2 & Capacidad de organización y planificación \\
\hline & 3 & Comunicación oral y escrita en lengua nativa \\
\hline & 4 & Conocimiento de una lengua extranjera \\
\hline & 5 & Conocimientos de informática relativos al ámbito de estudio \\
\hline & 6 & Utilizar las tecnologías de la información y la comunicación \\
\hline \multirow{6}{*}{ Personales } & 7 & Trabajo en equipo \\
\hline & 8 & Habilidades en las relaciones interpersonales \\
\hline & 9 & Razonamiento crítico \\
\hline & 10 & Compromiso ético \\
\hline & 11 & Trabajar en equipo con otros docentes \\
\hline & 12 & Afrontar los deberes y los dilemas éticos de la profesión \\
\hline \multirow{6}{*}{ Sistémicas } & 13 & Aprendizaje autónomo \\
\hline & 14 & Adaptación a situaciones nuevas \\
\hline & 15 & Creatividad \\
\hline & 16 & Organizar la propia formación continua \\
\hline & 17 & Organizar y animar situaciones de aprendizaje \\
\hline & 18 & Gestionar la progresión de los aprendizajes \\
\hline
\end{tabular}




\subsection{PROCEDIMIENTO}

La recogida de datos se realizó mediante un procedimiento aleatorio simple, durante los meses de marzo y abril del curso académico 2014/15. El cuestionario se aplicó de forma online para proporcionar el fácil acceso, sobre todo en el grupo de los egresados que ya presentan una menor vinculación a la universidad, aumentar la participación y facilitar la recogida de la información. Se garantizó en todo momento el anonimato de los participantes.

\subsection{ANÁLISIS}

En primer lugar, para someter a contraste la estructura factorial del instrumento se realizó el AFC (Análisis Factorial Confirmatorio) de la escala. Se han realizado los análisis estadísticos descriptivos básicos y se ha calculado la fiabilidad de la escala (Alfa de Cronbach .93), teniendo en cuenta las recomendaciones de $\mathrm{Hu}$ y Bentler (1999), en las cuales un modelo ajusta adecuadamente atendiendo a los índices RMSEA (Root Mean Square Error of Approximation), SRMR (Standardized Root Mean Square Residual), cuyos valores recomendables son $<.08$, los índices NNFI (Non-Normed Fit Index), IFI (Incremental Fit Index) y CFI (Comparative Fit Index), cuyos valores recomendables son > .95 (Jöreskog \& Sörbom, 1993). Como método de estimación, se utilizó el de máxima verosimilitud robusta que permite utilizar correlaciones policóricas, ya que su uso es más idóneo en variables con las características señaladas, de altos índices de normalidad y curtosis multivariante, y con una clara naturaleza ordinal (Flora \& Curran, 2004).

En segundo lugar, se realizó un análisis de varianza (ANOVA) con el fin de analizar las diferencias existentes entre los grupos y profundizar en aquellos grupos que presentaban diferencias estadísticamente significativas. Se eligió el método de Games-Howell para los tamaños muestrales diferentes.

Para realizar los análisis señalados, los programas informáticos utilizados fueron el SPSS 22 para los análisis de fiabilidad y la versión 6.2 del programa estadístico EQS para los análisis factoriales confirmatorios.

\section{RESULTADOS}

Los resultados del AFC nos muestran una solución factorial con cuatro factores (competencias instrumentales, competencias personales y competencias sistémicas) correlacionados con un ajuste bastante optimo, con valores de $\mathrm{x}^{2}$ de Satorra Bentler =2025.18; p=0.00; NNFI=.95; CFI=.96; IFI=.96; RMSA=.08; SRMR=.06. El AFC se realizó mediante una estimación robusta de máxima verosimilitud (MLR) porque la normalidad y curtosis multivariante son muy altas, de hecho, el valor del Coeficiente de Mardia es de 78.79.

En relación al primer objetivo, y para conocer cuáles son las competencias más desarrolladas desde el punto de vista de los participantes, podemos observar (Tabla 2) que en el grupo de los estudiantes la capacidad para trabajar en equipo es la competencia que presenta un valor más alto (3.27), seguida de la capacidad para aprender de forma autónoma (2.86). Sin embargo, este grupo se considera menos capacitado para hablar en una lengua extranjera (1.27) y para utilizar medios informáticos y tecnológicos, con un valor de 1.78. 
También en los docentes, la capacidad para trabajar en equipo es la competencia que presenta un valor más alto a las demás, al igual que los estudiantes (3.12). A continuación, la competencia más valorada por los docentes es la capacidad de razonamiento crítico (3.08). Asimismo, los docentes, al igual que los estudiantes, consideran que la capacidad menos desarrollada es la habilidad para hablar en otra lengua distinta a la nativa, con un valor de 1.07 .

El grupo de los egresados también presenta el mayor valor (3.34) en la competencia para trabajar en equipo, y el valor menor en la competencia en los conocimientos sobre una lengua extranjera (1.12), al igual que los estudiantes y los docentes. No obstante este grupo se considera muy capacitado, después del trabajo en equipo, para el desarrollo de habilidades y relaciones interpersonales (3.00).

Tabla 2. Estadísticos descriptivos de la escala

\begin{tabular}{|c|c|c|c|c|c|c|}
\hline \multirow{2}{*}{ Ítems } & \multicolumn{2}{|c|}{ Estudiantes } & \multicolumn{2}{c|}{ Docentes } & \multicolumn{2}{c|}{ Egresados } \\
\cline { 2 - 7 } & $\mathrm{N}$ & $\mathrm{M}$ & $\mathrm{N}$ & $\mathrm{M}$ & $\mathrm{N}$ & $\mathrm{M}$ \\
\hline 1 & 1231 & 2.40 & 344 & 2.97 & 487 & 2.57 \\
\hline 2 & 1230 & 2.63 & 345 & 2.85 & 481 & 2.81 \\
\hline 3 & 1226 & 2.48 & 341 & 2.72 & 488 & 2.64 \\
\hline 4 & 1223 & 1.27 & 341 & 1.07 & 488 & 1.12 \\
\hline 5 & 1231 & 1.78 & 344 & 2.03 & 490 & 1.86 \\
\hline 6 & 1232 & 2.26 & 348 & 2.57 & 484 & 2.30 \\
\hline 7 & 1233 & 3.27 & 345 & 3.12 & 490 & 3.34 \\
\hline 8 & 1230 & 2.83 & 344 & 2.90 & 489 & 3.00 \\
\hline 9 & 1232 & 2.69 & 345 & 3.08 & 488 & 2.84 \\
\hline 10 & 1229 & 2.55 & 344 & 2.83 & 489 & 2.75 \\
\hline 11 & 1233 & 2.48 & 347 & 2.27 & 490 & 2.66 \\
\hline 12 & 1232 & 2.16 & 347 & 2.34 & 490 & 2.26 \\
\hline 13 & 1232 & 2.86 & 345 & 2.92 & 482 & 2.92 \\
\hline 14 & 1232 & 2.63 & 345 & 2.77 & 486 & 2.84 \\
\hline 15 & 1229 & 2.61 & 344 & 2.77 & 491 & 2.75 \\
\hline 16 & 1231 & 2.32 & 346 & 2.09 & 489 & 2.46 \\
\hline 17 & 1232 & 2.50 & 348 & 2.57 & 489 & 2.74 \\
\hline 18 & 1233 & 2.40 & 349 & 2.47 & 488 & 2.64 \\
\hline & & & & & & \\
\hline
\end{tabular}

Posteriormente y en relación al segundo objetivo, realizamos análisis descriptivos de cada dimensión de la escala (instrumental, personal y sistémica) para comprobar cuál es el conjunto de competencias más desarrolladas en todos los grupos muestrales. En la Tabla 3 se puede apreciar que, con relación a las tres dimensiones, el valor más alto en todos los 
grupos (estudiantes, docentes y egresados) se manifiesta en las competencias personales, seguido de las sistémicas con un valor de 2.73 en los egresados, 2.60 en los docentes y 2.55 en los estudiantes. Asimismo, podemos observar que el nivel de capacitación en las competencias instrumentales es mayor en los docentes (2.37) y menor en los estudiantes (2.13). En las competencias personales el mayor valor lo presentan los egresados con 2.81, seguidos de los docentes con 2.76 y por último los estudiantes (2.66). En las competencias sistémicas el nivel de capacitación más alto lo presentan, nuevamente, los egresados, con un valor de 2.73 , frente a los estudiantes que consideran que su nivel adquirido en estas competencias es mucho menor (2.55).

Tabla 3. Estadísticos descriptivos por dimensiones de la escala

\begin{tabular}{|l|c|c|c|c|c|c|}
\hline \multirow{2}{*}{ Dimensión } & \multicolumn{2}{|c|}{ Docentes } & \multicolumn{2}{c|}{ Estudiantes } & \multicolumn{2}{c|}{ Egresados } \\
\cline { 2 - 7 } & $\mathrm{N}$ & $\mathrm{M}$ & $\mathrm{N}$ & $\mathrm{M}$ & $\mathrm{N}$ & $\mathrm{M}$ \\
\hline Instrumental & 337 & 2.37 & 1.212 & 2.13 & 463 & 2.22 \\
\hline Personal & 341 & 2.76 & 1.224 & 2.66 & 482 & 2.81 \\
\hline Sistémica & 339 & 2.60 & 1.226 & 2.55 & 473 & 2.73 \\
\hline
\end{tabular}

Para el tercer objetivo, en la Tabla 4 también observamos que existen diferencias significativas $(\mathrm{p}>.05)$ en los tres grupos en el conjunto del cuestionario. Especialmente, entre el grupo de estudiantes y egresados se establecen diferencias en las competencias personales y las competencias sistémicas. Entre el grupo de estudiantes y docentes encontramos únicamente diferencias en las competencias instrumentales. Finalmente, entre el grupo de egresados y docentes las diferencias se establecen en las competencias sistémicas.

Tabla 4. ANOVA en los tres grupos en cada una de las dimensiones de la escala

\begin{tabular}{|c|c|c|c|c|c|}
\hline Dimensión & G & $\mathrm{gl}$ & $\mathrm{F}$ & $\mathrm{P}$ & G-H \\
\hline \multirow{3}{*}{ Instrumental } & Estudiantes & \multirow{3}{*}{$\begin{array}{c}2 \\
2011\end{array}$} & 15.89 & $>.00$ & $\mathrm{Es} \neq \mathrm{D}$ \\
\hline & Egresados & & & & \\
\hline & Docentes & & & & \\
\hline \multirow{3}{*}{ Personal } & Estudiantes & \multirow{3}{*}{$\begin{array}{c}2 \\
2046\end{array}$} & 8.21 & $>.00$ & $\mathrm{Eg} \neq \mathrm{Es}$ \\
\hline & Egresados & & & & \\
\hline & Docentes & & & & \\
\hline \multirow{3}{*}{ Sistémica } & Estudiantes & \multirow{3}{*}{$\begin{array}{c}2 \\
2037\end{array}$} & 11.53 & $>.00$ & $\mathrm{Es} \neq \mathrm{Eg}$ \\
\hline & Egresados & & & $>.03$ & $E g \neq D$ \\
\hline & Docentes & & & & \\
\hline
\end{tabular}

La diferencia de medias es significativa al nivel .05 


\section{CONCLUSIONES}

En los resultados obtenidos, hemos podido comprobar que en el grupo de las competencias personales el trabajo en equipo es una de las competencias más valorada por los tres grupos, es decir que en general se sienten bastante capacitados para desarrollar esta habilidad o destreza. En los títulos de educación el aprendizaje cooperativo es una de las estrategias metodológicas más utilizadas (Goikoetxea \& Pascual, 2002), puede ser esta una variable que esté contribuyendo a la buena percepción de nuestros participantes.

Por otro lado, los resultados también nos demuestran que las competencias instrumentales, tanto en el grupo de estudiantes como el grupo de egresados y docentes, son las percibidas como menos desarrolladas, y dentro de este grupo la capacidad para hablar en otro idioma y la competencia tecnológica son las que presentan valores más bajos.

Del mismo modo, en el grupo de los docentes es donde existe una menor valoración con respecto al conocimiento de una lengua extranjera. La formación inicial del profesorado tiene, en este sentido, una importancia fundamental para el desarrollo de las competencias bilingües. No cabe duda de que son numerosos los esfuerzos realizados por parte del profesorado, generalmente con resultados positivos (Lahuerta, 2015; Lova, Bolarín \& Porto, 2012). Sin embargo, son numerosos los docentes que también manifiestan sus preocupaciones acerca de las competencias bilingües necesarias para el desarrollo de su docencia en el marco de un sistema de educación de calidad (Fernández \& González, 2015; Rubio \& Hermosín, 2010). El desarrollo de la competencia bilingüe, a su vez, es una propuesta cuya relevancia no queda limitada simplemente a la mejora del nivel de lengua en otro idioma, ni siquiera a la adquisición de unas destrezas concretas en el aprendizaje de esa lengua, sino a que requiere desarrollar las competencias para "enseñar y aprender en un idioma extranjero". Por lo tanto, y coincidiendo con Ramos (2007), no se trata sencillamente de formación lingüística, sino también de formación pedagógica y didáctica.

En la introducción de este trabajo, Bartram y Roe (2005) señalaban la necesidad de la formación del profesorado en competencias tecnológicas debido a que las Tecnologías de la Información y la Comunicación (TIC) tienen una importancia indiscutible en el ámbito académico y social. Hasta el día de hoy se les ha otorgado un lugar privilegiado en las relaciones sociales y en la formas de interactuar con el conocimiento, por ello este hecho no puede permanecer ajeno la Formación Inicial del Profesorado, que tiene la responsabilidad de brindar oportunidades para que los futuros maestros, en un diálogo entre las dimensiones teoría y práctica, desarrollen una base de conocimientos que les permita insertar su labor en el contexto de la sociedad del conocimiento (Martínez, Leite \& Monteiro, 2016). En este trabajo hemos podido comprobar que el desarrollo del grupo de habilidades y destrezas relacionadas con este grupo de competencias instrumentales demanda una mayor atención, en lo que a formación se refiere. Ser competente digitalmente y dominar las tecnologías requiere esfuerzos a nivel personal e institucional, porque coincidiendo con Paraskeva y Oliveira (2008), se puede afirmar que no basta con saturar de aparatos las aulas, sino que es necesario un cambio profundo en las concepciones que conforman las bases del sistema educativo. Es necesario comprender que existen otras dinámicas sociales que se resisten dentro de las aulas y que determinan nuevos modos de interactuar con el saber, que los estudiantes necesitan conocer, reconocer y participar para poder diseñar situaciones de enseñanza contextualizadas a los tiempos en los que nos encontramos. 
Las autoras de este trabajo consideran que las conclusiones extraídas del mismo pueden contribuir a la revisión de los planes de estudios de las titulaciones de educación, para la elaboración de políticas que promuevan el desarrollo de competencias en el dominio de una segunda lengua y de las tecnologías en la Formación Inicial del Profesorado.

Entre las limitaciones que se pueden aplicar a este trabajo se encuentra la imposibilidad de extraer conclusiones claras en la comparación de diferencias entre resultados del mismo grupo poblacional, ya sea estudiantes, egresados o profesorado, debido a la necesidad de considerar otras variables que puedan estar influyendo en los resultados. En este sentido, también debemos considerar las diferencias entre los diferentes grupos, estudiantes, egresados y docentes, con relación al desarrollo de determinadas competencias. Por ejemplo, no tendrá la misma percepción un estudiante, que un egresado y un profesor en competencias instrumentales, fundamentalmente porque el estudiante aún no ha concluido su formación, y en competencias personales y sistémicas, porque el profesor y el egresado habrán tenido que desarrollar habilidades relacionadas con su formación continua y su proyección laboral, entre otras.

Otra limitación que encontramos es el hecho de que los resultados obtenidos se basan, fundamentalmente, en la percepción de los participantes. Por lo tanto, sería conveniente realizar estudios posteriores con un análisis más específico de los resultados teniendo en cuenta otras variables de estudio.

Como futuras líneas de investigación se propone contemplar otras variables de tipo geográfico y de institución, analizar el contexto y los planes de estudio de las universidades participantes para comprobar de qué forma afectan a la Formación Inicial del Profesorado.

\section{REFERENCIAS BIBLIOGRÁFICAS}

Bartram, D., \& Roe, R. (2005). Definition and assessment of competences in the context of the European Diploma in Psychology. European Psychologist, 10, 93-102.

Elen, J., Clarebout, G., Léonard, R., \& Lowick, J. (2007). Student-centered and teacher-centred learning environments: what students think. Teaching in Higher Education, 12(1), 105-117.

Fernández, A., \& González, X.A. (2015). Teacher Satisfaction Concerning the Implementation of Bilingual Programmes in a Spanish University. Porta Linguarum, 23, 93-108.

Flora D.B., \& Curra, P.J. (2004). An Empirical Evaluation of Alternative Methods of Estimation for Confirmatory Factor Analysis with Ordinal Data. Psychological Methods, 9, 466-491. doi:10.1037/1082-989X.9.4.466

Goikoetxea, E., \& Pascual G. (2002). Aprendizaje cooperativo: bases teóricas y hallazgos empíricos que explican su eficacia. Educación XXI, 5, 227-247. doi:10.5944/educxx1.5.1.392

González, J., \& Wagenaar, R. (2003). Tuning Educational Structures in Europe I. Informe Final. Bilbao: Universidad de Deusto. Recuperado de http://tuningacademy.org/wp-content/ uploads/2014/02/TuningEUI_Final-Report_SP.pdf

Hu, L., \& Bentler, P. M. (1999). Cutoff criteria for fit indexes in covariance structure analysis: Conventional criteria versus new alternatives. Structural Equation Modeling. A Multidisciplinary Journal, 6, 1-55. doi:10.1080/10705519909540118

Jöreskog, K. G., \& Sörbom, D. (1993). LISREL 8: Structural equation modeling with the SIMPLIS command language. Hillsdale, NJ: Lawrence Erlbaum Associates Publishers.

Lahuerta, A. C. (2015). The Written Competence of Spanish Secondary Education Students in Bilingual and Non-Bilingual Programs. Porta Linguarum, 24, 7-61.

Le Boterf, G. (2001). Construire les compétences individuelles et collectives (2éd.). Paris: Éditions 
d'Organisation.

Lova, M., Bolarín, M. J., \& Porto, M. (2012). Programas bilingües en Educación Primaria: Valoraciones de docentes. Porta Linguarum, 20, 253-268.

Martínez, L.F., Castejón, F.J., \& Santos, M.L. (2012). Diferentes percepciones sobre evaluación formativa entre profesorado y alumnado en formación inicial en educación. Revista Electrónica Interuniversitaria de Formación del Profesorado, 15(4), 57-67.

Martínez, R., Leite, C., \& Monteiro, A. (2016). TIC y formación inicial de maestros: oportunidades y problemas desde la perspectiva de estudiantes. Cuadernos de Investigación Educativa, 7(1), 69-92. doi:10.18861/cied.2016.7.1.2577

Muros, B., \& Luis-Pascual, J.C. (2012). Aprendizaje, capacidades cognitivas y evaluación formativa en formación inicial del profesorado. Revista de Ciencias de la Educación, 230, 171-182.

Novak, J. D. (2003). The Promise of New Ideas and New Technology for Improving Teaching and Learning. Cell Biology Education, 2, 122-132.

Paraskeva, J., \& Oliveira, L. (2008). Currículo e Tecnologia Educativa. Mangualde: Edições Pedago.

Perrenoud, P. (1997). Construiré des compétences des l'école (3éd.). París: Editions Sociales Francaises.

Prieto, L. (Coord.). (2008). La enseñanza universitaria centrada en el aprendizaje. Barcelona: Octaedro/ICE-UB.

Ramos, F. (2007). Programas bilingües y formación de profesores en Andalucía. Revista Iberoamericana de Educación, 44, 133-146.

Rodríguez-Esteban, A. (2007). Las competencias en el Espacio Europeo de Educación Superior: Tipologías. Humanismo y Trabajo Social, 6, 83-91.

Rodríguez-Esteban, A., \& Vieira M.J. (2009). La formación en competencias en la universidad: un estudio empírico sobre su tipología. Revista de Investigación Educativa, 27(1), $27-47$.

Rogoff, B. (1999). Cognitive Development through Social Interaction: Vygotsky and Piaget. In P. Murphy (Coord.), Learners, Learning and Assessment (pp. 69-82). London: Paul Chapman Publishing.

Rubio, F. D., \& Hermosín, M. J. (2010). Implantación de un programa de plurilingüismo en el espacio europeo de educación superior: Análisis de contexto y detección de necesidades. Revista de Educación, 12, 107-128.

Ruíz-Gallardo, J.R., Ruíz, E., \& Ureña, N. (2013). La evaluación en la Formación Inicial del Profesorado: Qué creemos hacer y qué perciben los alumnos. Cultura, ciencia y deporte, 8(22), 17-29. doi:10.12800/ccd.v8i22.220

Rust, C., Price, M., \& O' Donovan, B. (2003). Improving students' learning by developing their understanding of assessment criteria and processes. Assessment and Evaluation in Higher Education, 28(2), 147-164.

Struyven, K., Dochy, F., \& Janssens, S. (2010). ‘Teach as you preach': the effects of student-centred versus lecture-based teaching on student teachers approaches to Teaching. European Journal of Teacher Education, 33(1), 43-64.

Villa, A., \& García Olalla, A. (2009). Competencias docentes en el ámbito universitario. Bilbao: Universidad de Deusto.

Whiddett, S., \& Hollyforde, S. (2003). The competencies handbook. London: CIPD.

Zabala, A., \& Arnau, L. (2008). Once ideas clave. Cómo aprender y enseñar competencias. Barcelona: Graó.

Zabalza, M.A. (2011). Evaluación de los planes de formación docente de las universidades. Educar, 47(1), 181-197. doi:10.5565/rev/educar.77 
